\title{
Gully geometry: what are we measuring?
}

\author{
J. Casalí, R. Giménez, and M. A. Campo-Bescós \\ Department of Projects and Rural Engineering, Public University of Navarre, Los Olivos Building, \\ 31006 Pamplona, Spain \\ Correspondence to: J. Casalí (jcs@ unavarra.es)
}

Received: 10 March 2015 - Published in SOIL Discuss.: 31 March 2015

Revised: 05 June 2015 - Accepted: 26 June 2015 - Published: 10 July 2015

\begin{abstract}
Much of the research on (ephemeral) gully erosion comprises the determination of the geometry of these eroded channels, especially their width and depth. This is not a simple task due to uncertainty generated by the wide range of variability in gully cross section shapes found in the field. However, in the literature, this uncertainty is not recognized so that no criteria for their measurement are indicated. The aim of this work is to make researchers aware of the ambiguity that arises when characterizing the geometry of an ephemeral gully and similar eroded channels. In addition, a measurement protocol is proposed with the ultimate goal of pooling criteria in future works. It is suggested that the geometry of a gully could be characterized through its mean equivalent width and mean equivalent depth, which, together with its length, define an "equivalent prismatic gully" (EPG). The latter would facilitate the comparison between different gullies.
\end{abstract}

\section{Introduction}

The classic forms of water erosion are caused by nonconcentrated or laminar flow and concentrated flow; in the latter, a distinction is made between rill and gully erosion (Hutchinson and Pritchard, 1976). Rill erosion occurs in the form of numerous channels of a few centimetres in depth, distributed uniformly and randomly over sloping land (Soil Science Society of America, 2015) and easily obliterated by conventional tillage (Hutchinson and Pritchard, 1976). Furthermore, permanent gullies are distinguished from ephemeral ones (Foster, 1986; Thorne et al., 1986; Casalí et al., 1999). Permanent gullies are erosion channels which are too large to be eliminated by conventional tillage (Soil Science Society of America, 2015). Ephemeral gullies present in agricultural soils - are, like rills, small enough for it to be possible to eliminate them by traditional tillage (Soil Science Society of America, 2015), hence their being qualified as ephemeral. However, when they form again, they tend to appear in the same places, contrary to what is observed in rills. This is explained by the fact that the ephemeral gullies are formed in the thalweg, which constitutes the confluence of two opposing slopes, a fact which conditions the trajectory of the runoff. Rills, however, occur entirely on one single slope (Casalí et al., 1999); their formation is, therefore, mainly subject to the high spatial variability of intrinsic factors of the soil (structural stability, hydraulic conductivity, etc.) and of its tillage.

The objectives of a large number of works on gully erosion have been the estimation of the spatial and/or temporal evolution of a gully or a network of gullies under different conditions (e.g. climate, land use) (e.g. Casalí et al., 2006; Gabet and Bookter, 2008; Campo-Bescós et al., 2013). For that purpose, as a first step, a morphological characterization of these channels is made. The most frequent way to do so is by measuring their width and depth and the ratio between both parameters (e.g. Giménez et al., 2009); their typology is also studied (for example, whether their cross section presents a particular shape, such as a $\mathrm{U}$ or a $\mathrm{V}$ ). If the measurement of the length of the gully is added to this, it may be possible to determine its volume (eroded soil).

Consequently, for a precise description of the geometry of a gully, the correct determination of its width is a key factor. This is not always an easy task, especially when faced with cross sections with intricate shapes and diffuse limits. However, in the numerous scientific works on the subject, no uncertainty whatever is expressed regarding this measurement, and the criteria followed in the procedure are not specified. We believe that, as a general rule, it is assumed that a gully's 

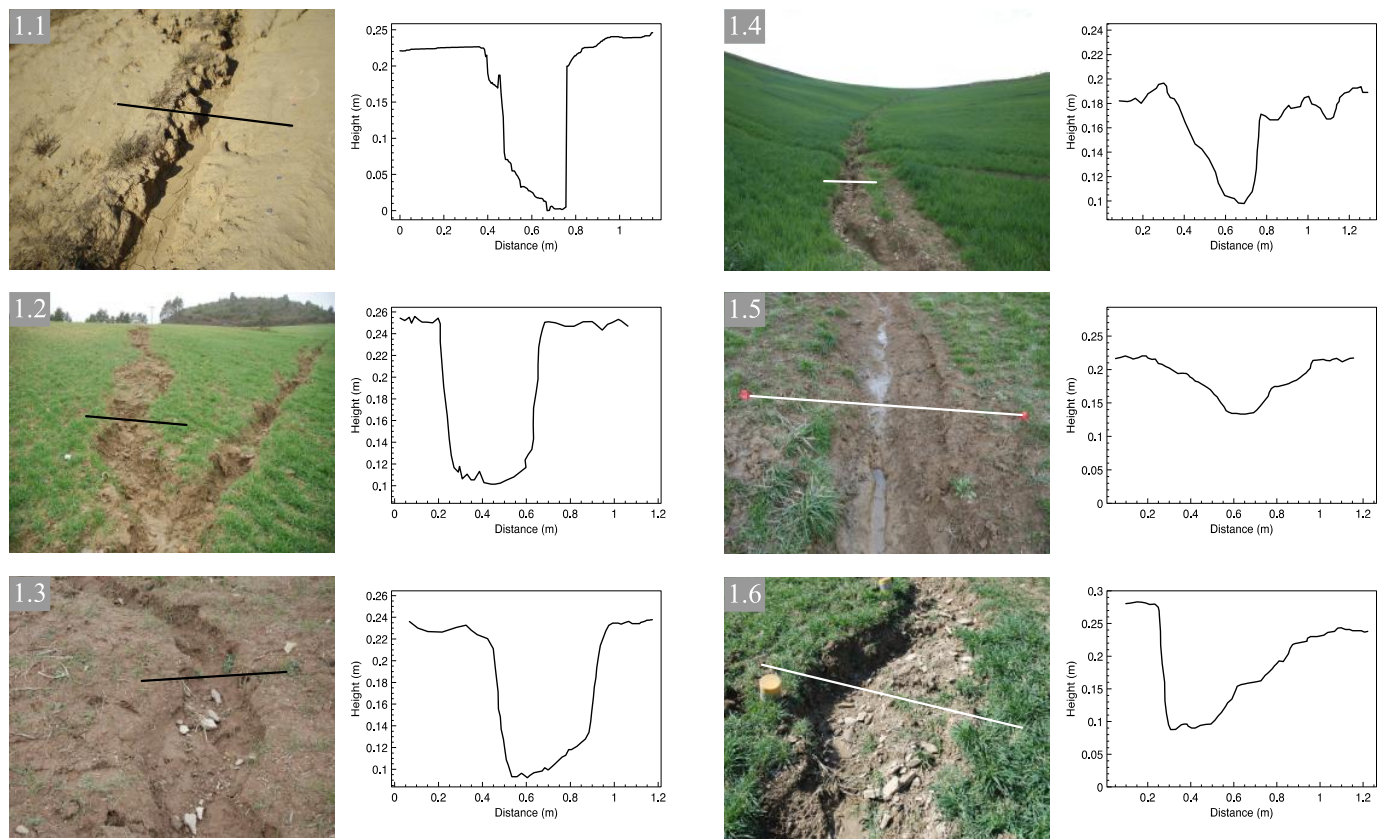

Figure 1. Examples of cross sections of typical ephemeral gullies (Navarre, Spain).

width is defined by the imaginary line whose ends are located at the points of the two banks where an abrupt change in slope is manifested. This criterion is followed both in direct measurements in situ and in indirect ones taken from digital elevation models and mathematic algorithms ad hoc (e.g. Evans and Lindsay, 2010; Parker et al., 2012; Castillo et al., 2014). However, this procedure, at first sight reasonable and unquestionable, raises two objections. First, there is the presence of more than one point of slope inflection in one or both banks. Second, even when only one visible inflection point is presented on the slope of each bank - with the width of the channel thus being clearly defined - this also poses a question: do the limits of this channel, defined in this way, really correspond to the transversal limits of the erosive process which gave rise to the gully? Only by knowing the topography of the land in the moments before the formation of the gully can one answer that question with any certainty.

On the other hand, the width of a gully defines the upper limit of its cross section, therefore conditioning the subsequent determination of the depth of that channel. Furthermore, in this latter measurement (depth of the gully), another important ambiguity is added, i.e. the determination of the lower limit of the cross section (channel bed). This latter limit is usually located - in our belief - at the lowest point of the cross section, which is questionable in beds with a highly irregular cross-sectional profile. Nevertheless, the difficulty inherent in measuring a gully depth is not usually emphasized in the literature either.

In short, the lack of any protocol or universal criterion in determining the geometry of gullies would then cause a particular uncertainty when comparing the experimental results

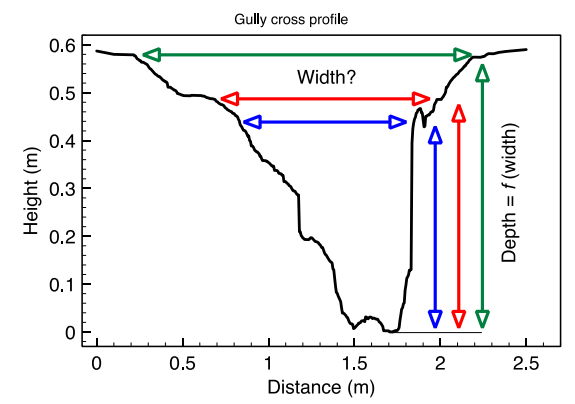

Figure 2. Uncertainty in the determination of a width in a cross section of a gully (real example). Arrows show different cross section widths and their corresponding depths (same colour). See Sect. 2 for details.

obtained by different researchers, for example, erosion rate values.

In this work it is sought to alert the scientific community to the uncertainties of which researchers are often unaware and which are triggered when characterizing the geometry of an ephemeral gully; for this purpose some examples of real cases will be shown. In addition, a measurement protocol is proposed, with the ultimate aim of pooling criteria in future works and experimentation. Although the criteria are proposed for ephemeral gullies, they would equally apply to similar erosion channels. 

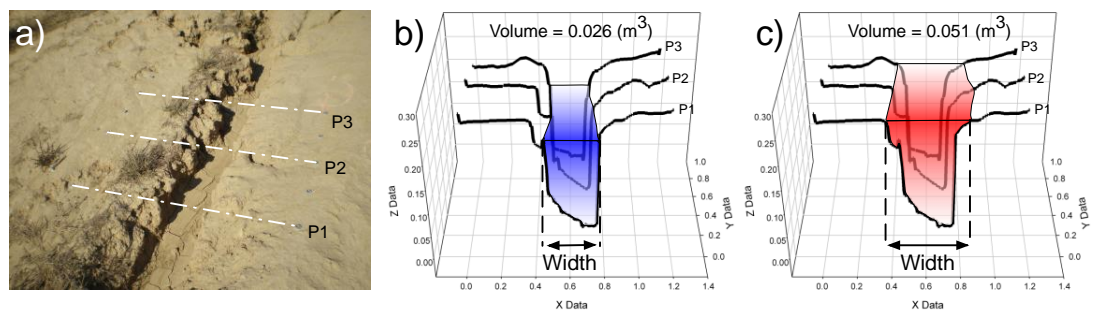

Figure 3. Illustration of the effect that the criterion followed to determine the cross section width exerts on the computed volume of a gully reach. (a) Selected gully reach and location of the three cross sections used for calculating the volume of the reach (P1, P2 and P3); the distance between cross sections is known. (b) Calculated eroded volume (in blue) when considering one possible criterion for defining the gully cross section widths. (c) Calculated eroded volume (in red) when considering a different possible criterion for defining the gully cross section widths.
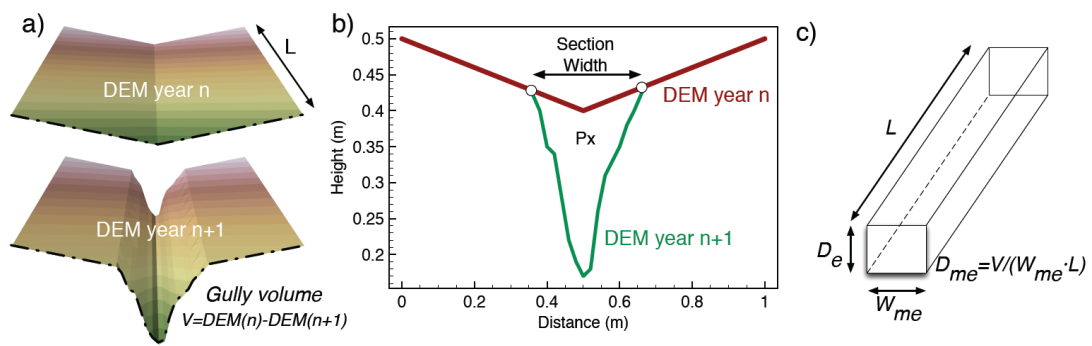

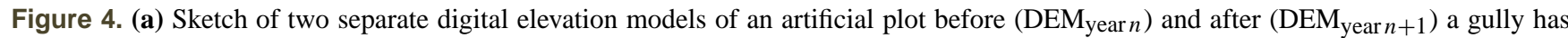
been formed in the plot thalweg; (b) sketch of cross section area depicted at any point $x$ along the longitudinal axis of the gully; (c) equivalent prismatic gully (EPG). See Sect. 3 for details.

\section{Uncertainties in measuring the width and depth of a gully}

Researchers, especially those new to the subject, when confronted with the measurement of gully geometry, assume that the limits of the erosion channel will present themselves in the field as being clearly defined, and, in fact, this is often true (see Fig. 1.1-1.3). However, on many occasions this is not the case (Fig. 1.4-1.6). It is therefore possible that a clear break in the slope of one of the banks (Fig. 1.6) or in both of them (Fig. 1.5) may not be noticed. Another possible ambiguity - independent of or additional to the previous one - is that which arises when both banks of the channel are uneven (Fig. 1.4, 1.6). This means that determining a single height value to trace an imaginary horizontal line between both banks is highly subjective. The length of this line should be understood as defining the width of the cross section being measured.

By contrast, when defining the depth of a gully, the lower limit of the cross section is usually well defined by the lowest point of the bed (see Fig. 1.2). However, what usually happens is that the location of this limit is also controversial, as can be seen in the cross sections in Fig. 1.1 and 1.3, where it is not clear whether this limit would really be represented by the lower height of the bed.

An incorrect determination of the width and/or depth of a certain gully may cause (important) errors in the determi- nation of its volume, i.e. in the estimation of the eroded soil (Figs. 2 and 3). The magnitude of this potential experimental error would be less obvious, and even underestimated, if we analysed the cross sections individually (Fig. 2). However, an overall review of all the sections making up the gully being studied would give a better assessment of this measurement error. Figure 3 aims to illustrate the effect that the criterion followed to determine the cross section width exerts on the computed volume of a gully reach. A real gully reach was selected and three cross sections were used for calculating the volume of the reach (P1, P2 and P3) (Fig. 3a), the distance between cross sections being known. First, the eroded volume was calculated considering one possible criterion for defining the gully cross section width (in blue, Fig. 3b). Then the eroded soil was calculated again but considering a different possible criterion for defining the gully cross section widths (in red, Fig. 3b). The difference in the calculated volume is remarkable, increasing by $96 \%$ from option b to option c. Figure 3 is just one example illustrating (i) the great differences in volumes that can be obtained in fixing the gully widths arbitrarily; (ii) the error that can be generated and (iii) the necessity of establishing rigorous and objective criteria and protocols. The purpose of Fig. 3 is similar to Fig. 2, the latter depicting the effect of the uncertainty in the determination of width in a single cross section of a gully. 


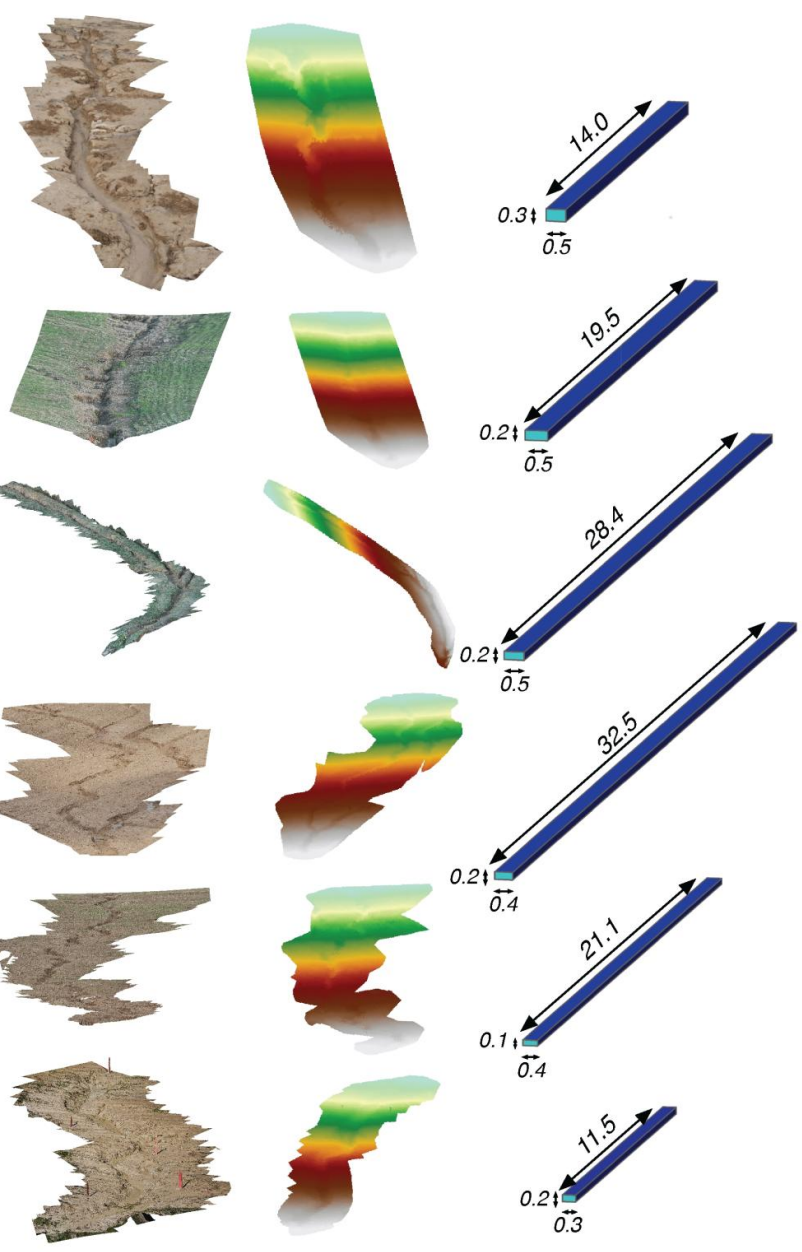

Figure 5. (a) Pictures of ephemeral gullies of different shapes (Navarre, Spain); (b) digital elevation model $\left(\right.$ DEM $_{\text {year } n+1}$, see Fig. 4) of each gully; (c) equivalent prism of the gullies since there was no DEM available prior to the gully formation $\left(\mathrm{DEM}_{\mathrm{year} n}\right.$, see Fig. 4), the width was arbitrarily defined on the basis of abrupt changes at both gully banks (see Sect. 3)). It should be made clear that the geometry of the equivalent prisms could have changed (dramatically) if we had also taken the corresponding DEMyear $n$ into account. (Please note that lengths are given in metres.)

\section{Topographic definition of gully width, equivalent prismatic gully (EPG)}

Let us suppose that we have a detailed digital elevation model (DEM) of a gully whose geometry we wish to determine (Fig. 4a). Similarly, we would also have a DEM, not more than 1 year old, of the same area, but before the gully in question had formed. Remember that the cycle of the formation and obliteration of an ephemeral gully is conditioned by the periodicity (usually 1 year) of the agricultural tillage responsible for it. We shall call the DEM prior to the appearance of the gully $\mathrm{DEM}_{\text {year } n}$, whereas that of the following year - that is, with the gully $-\mathrm{DEM}_{\text {year } n+1}$ (Fig. 4a).
Let us imagine now that, at any point $x$ along the longitudinal axis of length $L$ of the gully, we draw a vertical plane $P_{x}$, perpendicular to that axis (Fig. 4b). If in this plane $P_{x}$ we subtract the $\mathrm{DEM}_{\text {year } n+1}$ from the $\mathrm{DEM}_{\text {year } n}$, we should obtain the eroded area or cross section of the gully (Fig. 4b). Now, the imaginary line which arises from joining the two points of the intersection of both DEMs would define, in turn, the width of the gully in that section $\left(P_{x}\right)$ (Fig. 4b). In the case of both points being uneven, a horizontal projection of the line should be considered. This same operation could be repeated at a multitude of other points $x i$ along the channel, thus obtaining the width value of each new section $\left(W_{i}\right)$. Finally, the average of the values $W_{i}$ would define the mean equivalent width of the whole gully, $W_{\text {me }}$. Those widths, determined thus, would undoubtedly be the true transversal limit of the erosion process which caused the gully in question.

If we now carry out the subtraction of both DEMs but on their entire surface, we should obtain the volume $V$ of the gully (Fig. 4a).

Furthermore, knowing $V$ and $W_{\text {me }}$, we could, in turn, determine a mean equivalent depth, $D_{\text {me }}$, expressed as

$D_{\mathrm{me}}=V /\left(W_{\mathrm{me}} L\right)$.

This depth value would be more representative of the whole gully than a value resulting from considering the minimum height of the bed as being the lower limit of the cross section (see above).

Finally, the gully could be represented as a rectangularbased prism ( $\left.W_{\mathrm{me}} D_{\mathrm{me}}\right)$ of a length $L$, which we call "equivalent prismatic gully" (EPG) (Figs. 4c and 5). This sort of normalization of the complex geometry of a particular gully - by means of its respective EPG - would permit, for example, a quick visual comparison of the individuals of a varied population of gullies (Fig. 5). It would thus be an interesting tool to incorporate into simulation models (e.g. AnnAGNPS, Gordon et al., 2007).

In effect, we believe that the concept of equivalent prismatic gully shows several benefits and applications. The principal one is that it permits the determination of the most important characteristics of a complete gully $\left(V, L, W_{\text {me }}\right.$ and $D_{\text {me }}$ ), using objective and repeatable criteria. Otherwise, there is the risk of assigning information from specific cross sections or reaches to the whole gully. Moreover, the gully properties $\left(V, L, W_{\text {me }}\right.$ and $\left.D_{\text {me }}\right)$, as defined here, can be incorporated into statistical analyses or similar studies in which many gullies are involved, using a common language, repeatable and comparable among different researchers. Furthermore, by using the concept of an equivalent prismatic gully, sets of complete gullies can easily be graphically represented, which enables a quick and explanatory visual comparison.

The width of a gully cross section, as defined in this paper, depends on the DEM pixel size and it depends on the type and size of the studied channel. Hengl (2006) concluded that, 
to prevent the loss of relevant information, the maximum pixel size must be the average of the minimum distances between sampling points. In the same way, Garbrecht and Martz (1994) fixed the pixel size to the size of the smallest distinguishable object. Additionally, the new methodologies available (terrestrial or aerial lidar, 3-D photo-reconstruction, etc.) provide very detailed information, which may be more than enough, in our opinion, for the purposes of these studies. However, these thresholds should be explored in future research.

\section{Conclusions}

In order to progress in gully erosion research, clear criteria to define and determine the key morphological characteristics of gullies and their related properties (such as volumes) are needed. In this paper, a new proposal for advancing towards that goal has been submitted. Thus, starting from a precise definition of the width of each gully cross section, the mean equivalent gully width and depth are defined, as is the equivalent prismatic gully. This approach permits the determination of the most important characteristics of a complete gully ( $V, L, W_{\mathrm{me}}$ and $D_{\mathrm{me}}$ ), using objective criteria. Moreover, the gully properties defined here can be incorporated into statistical analyses using a common language among different researchers. Furthermore, by using the EPG, sets of complete gullies can be easily graphically represented, which allows for an explanatory visual comparison. The definition of the width of each gully cross section assumes that the topography of the area before the gully appearance is known. This is, in fact, very infrequently so, so that a new line of research arises. In any case, we believe that the proposal is a considerable advance in the applied research on gullies because it allows one to standardize the definition and determination of the most important characteristics of these erosion forms.

Acknowledgements. This study was partly funded by the Spanish Ministry of Science and Innovation (project CGL2011-24336).

Edited by: E. Taguas

\section{References}

Campo-Bescós, M. A., Flores-Cervantes, J. H., Bras, R. L., Casalí, J., and Giráldez, J. V.: Evaluation of a gully headcut retreat model using multitemporal aerial photographs and digital elevation models, J. Geophys. Res.-Earth, 118, 1-15, 2013.

Casalí, J., López, J. J., and Giráldez, J. V.: Ephemeral gully erosion in southern Navarra (Spain), Catena, 36, 65-84, 1999.

Casalí, J., Loizu, J., Campo, M. A., De Santisteban, L. M., and Álvarez-Mozos, J.: Accuracy of methods for field assessment of rill and ephemeral gully erosion, Catena, 67, 128-138, 2006.

Castillo, C., Taguas, E. V., Zarco-Tejada, P., James, M. R., and Gómez, J. A.: The normalized topographic method: An automated procedure for gully mapping using GIS, Earth Surf. Proc. Land., 39, 2002-2015, 2014.

Evans, M. and Lindsay, J.: High resolution quantification of gully erosion in upland peatlands at the landscape scale, Earth Surf. Proc. Land., 35, 876-886, 2010.

Foster, G. R.: Understanding ephemeral gully erosion, in: Soil Conservation. Assessing the National Resources Inventory 2. Committee on Conservation Needs and Opportunities, Board of Agriculture, National Research Council, National Academy Press, Washington, 90-125, 1986.

Gabet, E. J. and Bookter, A.: A morphometric analysis of gullies scoured by post-fire progressively bulked debris flows in southwest Montana, USA, Geomorphology, 96, 298-309, 2008

Garbrecht, J. and Martz, L.: Grid size dependency of parameters extracted from digital elevation models, Comput. Geosci., 20, 8587, 1994.

Giménez, R., Marzolff, I., Campo, M. A., Seeger, M., Ries, J. B., Casalí, J., and Álvarez-Mozos, J.: Accuracy of high-resolution photogrammetric measurements of gullies with contrasting morphology, Earth Surf. Proc. Land., 34, 1915-1926, 2009.

Gordon, L. M., Bennett, S. J., Bingner, R. L., Theurer, F. D., and Alonso, C. V.: Simulating ephemeral gully erosion in AnnAGNPS, Transactions of the American Society of Agricultural and Biological Engineers, 50, 857-866, 2007.

Hengl, T.: Finding the right pixel size, Comput. Geosci., 32, 1298 1283, 2006.

Hutchinson, D. E. and Pritchard, H. W.: Resource conservation glossary, J. Soil Water Conserv., 31, 1-63, 1976.

Parker, C., Clifford, N. J., Thorne, C. R.: Automatic delineation of functional river reach boundaries for river research and applications, River Research and Applications, 28, 1708-1725, 2012.

Soil Science Society of America: Glossary of Soil Science Terms, Soils Science Society of America, Madison, WI, available at: https://www.soils.org/publications/soils-glossary\#, last access: 15 February 2015.

Thorne C. R., Zevenbergen, L. W., Grissinger, E. H., and Murphey, J. B.: Ephemeral gullies as sources of sediment, Proceedings of the Fourth Federal Interagency Sedimentation Conference, 1, 3.152-3.161, 1986. 